\title{
Enhanced Muscle Growth by Plasmid-Mediated Delivery of Myostatin Propeptide
}

\author{
Shengwei Hu, ${ }^{1}$ Chuangfu Chen, ${ }^{1}$ Jingliang Sheng, ${ }^{1}$ Yufang Sun, ${ }^{2}$ Xudong Cao, ${ }^{1}$ and Jun Qiao ${ }^{1}$ \\ ${ }^{1}$ College of Animal Science and Technology, Shihezi University, Shihezi 832002, China \\ ${ }^{2}$ Jiangsu Key Laboratory for Biodiversity and Biotechnology, College of Science, Nanjing Normal University, Nanjing 210046, China
}

Correspondence should be addressed to Chuangfu Chen, chencf1962@yahoo.com

Received 9 August 2009; Accepted 30 December 2009

Academic Editor: Matthew B. Wheeler

Copyright ( $) 2010$ Shengwei Hu et al. This is an open access article distributed under the Creative Commons Attribution License, which permits unrestricted use, distribution, and reproduction in any medium, provided the original work is properly cited.

\begin{abstract}
Myostatin is a member of the transforming growth factor beta (TGF- $\beta$ ) superfamily that functions as a negative regulator of skeletal muscle development and growth. Myostatin blockade therefore offers a strategy for promoting muscle growth in livestock production without resorting to genetic manipulation. In this report, we examined the effect of myostatin inhibition by plasmidmediated delivery of a mutant myostatin propeptide (MProD76A), a natural inhibitor of myostatin, on the growth performance of mice. A significant increase in skeletal muscle mass was observed after a single intramuscular injection of naked plasmid DNA encoding MProD76A into mice. Enhanced muscle growth occurred because of myofiber hypertrophy, but no cardiac muscle hypertrophy and organomegaly was observed in the mice after myostatin inhibition by plasmid-mediated MProD76A delivery. These results demonstrate a promising approach to enhancing muscle growth that warrants further investigation in domestic animals.
\end{abstract}

\section{Introduction}

Myostatin is a secreted protein that acts as a negative regulator of skeletal muscle mass [1]. Myostatin gene knockout mice have about a doubling of skeletal muscle weights throughout the body as a result of a combination of muscle fiber hyperplasia and hypertrophy [2]. Natural mutations of myostatin also cause double-muscling phenotype in cattle breeds [3-5], Texel sheep [6], dogs [7], and even humans [8]. These findings have suggested that agents capable of inhibiting myostatin activity may have applications for promoting muscle growth in livestock production.

To enhance growth performance of animal or livestock, various molecular strategies and traditional breeding techniques have been developed to regulate myostatin activity. Myostatin gene knockout mice and dominant-negative myostatin mice have a dramatic and widespread increase in skeletal muscle mass $[2,9]$, and so do transgenic mice $[10]$ and fish $[11,12]$ expressing higher levels of myostatin inhibitors. The JA16 neutralizing antibodies against myostatin have been shown to be capable of increasing muscle mass by $\approx 25 \%$ when administered to normal mice [13]. However, all these approaches used in the above studies are of too high cost, complex and impractical for the widespread applications in livestock production. Thus, developing a practical and efficacious strategy that can enhance livestock muscle growth by regulating myostatin activity becomes an important issue.

It is well known that the direct injection of plasmid DNA into muscle is simple, inexpensive, and safe nonviral gene transfer techniques in vivo [14]. Skeletal muscle can be transfected in vivo by direct plasmid DNA injection, where foreign genes can be expressed at significant levels for up to 19 month [15-18]. These characteristics make plasmid-mediated gene delivery an excellent candidate for agricultural application. In addition, the previous studies have demonstrated that mutant myostatin propeptide (MProD76A), a natural inhibitor of myostatin, causes a significant increase in muscle mass after it was injected into normal mice [19] or $m d x$ mice [20]. Thus, the combination of plasmid-mediated gene delivery techniques and MProD76A may be a promising strategy that can be widely applied to improve animals or livestock growth. 
In this report, we investigated the effect of myostatin inhibition on the growth performance of mice by plasmidmediated MProD76A delivery. For increasing transgene expression, bupivacaine was introduced as adjuvant. The bupivacaine has been shown to result in a 4-80-fold enhancement of plasmid gene expression in mice and rats [21]. A significant increase in body mass was observed after a single intramuscular injection of plasmid DNA (pCI-MProD76AEGFP) into the regenerating quadriceps muscle of mice. Moreover, we encountered no adverse effects resulting from plasmid delivery to inhibit myostatin activity.

\section{Materials and Methods}

2.1. Expression Plasmids and DNA Preparation. The mouse myostatin propeptide cDNA (bases 1-798, GenBank accession no. NM_010834) was generated by reverse-transcription PCR from Kunming mice muscle tissue. The forward and reverse primers utilized for myostatin propeptide were: $5^{\prime}$ GCCACCATGATGCAAAAACTGCAAATGTATG-3', 5' CGGGACCTCTTGGGTGTGTCTGTC-3'. The forward primer contained Kozak consensus sequence (GCCACC) at the translation initiation site. The propeptide gene was then cloned into pMD18-T vector (TaKaRa Biotech, Dalian) to form the pMD18-MPro. The mutation D76A was introduced in this plasmid by performing PCR-based sitedirected mutagenesis at nucleotide position 299 (counting from start codon atg). The mutated propeptide (amino acids 1-266) was inserted into the Sac I /BamH I sites of pEGFP-N1 (including a linker before EGFP gene) to generate MProD76A-EGFP fusion gene, and ultimately the MProD76A-EGFP fragment was cloned into the Xho I /Not I sites of pCIneo plasmid (Promega) to form pCIMProD76A-EGFP construct (Figure 1). The EGFP gene was cloned into the XhoI/NotIsites of pCIneo plasmid to form pCI-EGFP construct as control. Plasmids were amplified in Escherichia coli $\mathrm{DH} 5 \alpha$ and purified with an EndoFree Plasmid Giga Kit (Tiangen Biotech Co. Ltd, Beijing, China). All constructs were subjected to DNA sequence analysis to verify integrity of the constructs.

2.2. Cell Culture and In Vitro Gene Expression Analysis. C2C12 myoblasts were seeded $18 \mathrm{~h}$ prior to transfection at a density of $7.5 \times 10^{4}$ cells $/ \mathrm{mL}$ in 6-well dishes $(2 \mathrm{~mL})$ in growth medium (DMEM + glutamax containing $10 \%$ FBS and $0.5 \%$ gentamycin/ampicillin). C2C12 cells were tranfected with plasmid DNA $(2 \mu \mathrm{g})$ by using lipofectamine following the manufacturer's procedure. After transfection, the medium was replaced with differentiation medium (DMEM containing 5\% HS) to allow the cells to differentiate. After $72 \mathrm{~h}$ differentiation, cells were incubated in serum-free medium for an additional $48 \mathrm{~h}$. The conditioned media from each plasmid-transfected $\mathrm{C} 2 \mathrm{C} 12$ cells were harvested and stored at $-80^{\circ} \mathrm{C}$ until further expression analysis. The efficiency of transfection was estimated by EGFP fluorescence using an Olympus fluorescent microscope. Conditioned media from plasmid-transfected C2C12 cells were concentrated 15 -fold by lyophilization and used to detect secreted MProD76A-EGFP or EGFP by Western blotting. EGFP polyclonal antibodies raised against a bacterially expressed EGFP were used in the Western blotting which was performed as described previously [22].

2.3. Intramuscular Injection of Plasmid DNA in Mice. All experiments involving animals were approved by the Animal Care Committee of Shihezi University. Kunming male mice (Laboratory animal centre, Xinjiang, China) were housed in a room controlled for lighting (12-h light, 12-h dark cycle) and temperature $\left(22 \pm 2^{\circ} \mathrm{C}\right)$. The mice were allowed free access to chow and water. On day $-5,50 \mu \mathrm{l}$ of $0.75 \%$ bupivacaine hydrochloride was injected into the left quadriceps muscle of mice (4 weeks old) [21]. On day 0 , the mice were weighed and allocated to two groups, and the regenerating quadriceps muscle was injected with $100 \mu \mathrm{g}$ pCI-MProD76AEGFP or pCI-EGFP in $100 \mu \mathrm{l}$ PBS. The mice were weighed once every 5 days and killed 30 days later. Epididymal fat pad, heart, kidney, liver, and individual muscles from both sides of each mouse were carefully dissected, and the average weight was used for each tissue.

2.4. RT-PCR. The RT-PCR method was used to detect mRNA expression levels of the MProD76A-EGFP gene in vivo after intramuscular injection of pCI-MProD76AEGFP or pCI-EGFP. The injected quadriceps muscles were used for total RNA extraction following the manufacturer's procedure. cDNA was synthesized with the ThermoScript RT-PCR system (Invitrogen). The specific primers used for detection of MProD76A-EGFP were as follows: $5^{\prime}$-ACACCCAAGAGGTCCCG-3' and 5'-TTGAAGTCGATGCCCTTC$3^{\prime}$. The primers for amplification of $\beta$-actin were as follows: $5^{\prime}$-ATCACTATTGGCAACGAGCG-3' and $5^{\prime}$-ACTCATCGTACTCCTGCTTG-3'. The primers for amplification of EGFP were as follows: $5^{\prime}$-CCCGAAGGCTACGTCCAGGAG-3' and 5' -CGATGGGGGTGTTCTGCTGGTA-3'. The primers for amplification of endogenous myostatin were as follows: $5^{\prime}$-TCCGCATACTCATCTTGTGC-3' and 5' TGCTCTCACTTCCTTGCCT-3' . The products were cloned and subjected to sequence analysis.

2.5. MProD76A-EGFP Quantification in Serum. Blood samples were collected every week by retro-orbital puncture of anesthetized mice. Quantification of MProD76A-EGFP in these samples was essentially performed as described previously [23], but modified as follows. A known quantity of standard EGFP was used to generate standard curves for fluorescence intensity and protein quantity. The standard EGFP was suspended in either deionized water or serum from uninjected mice to evaluate the effects of varying conditions on fluorescence. The MProD76A-EGFP levels were quantified using the standard curve equation from the known EGFP quantity. The values were normalized by subtracting the 535-nm values of the uninjected control samples. All EGFP fluorescence intensity assay was performed with a PerkinElmer Victor ${ }^{3} 1420$ multilabel counter at $535 \mathrm{~nm}$ in counts per second. 


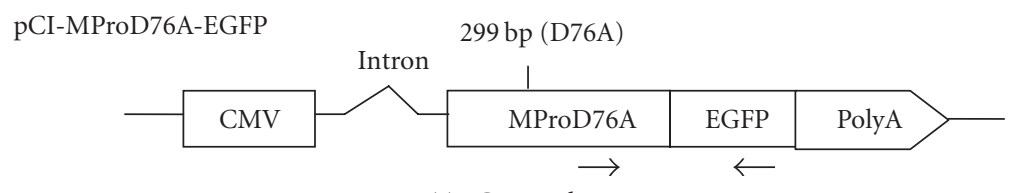

pCI-EGFP

RT-PCR: $510 \mathrm{bp}$

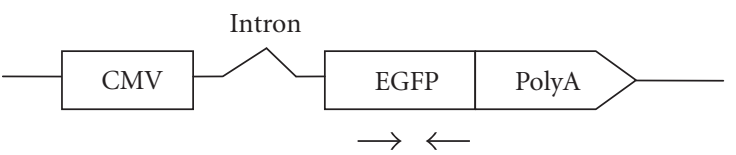

RT-PCR: $300 \mathrm{bp}$

FIGURE 1: Schematic illustration representing plasmids used in this study. The mutant myostatin propeptide (MProD76A) gene was cloned into the pCIneo plasmid containing a CMV promoter, chimeric intron and simian virus 40 (SV40) poly (A) sequence. The $5^{\prime}$ primer $(\rightarrow)$ and the $3^{\prime}$ primer $(\leftarrow)$ are in the coding area of MProD76A and EGFP, respectively. A 510-bp band indicated mRNA expression of MProD76AEGFP. A 300-bp band indicated mRNA expression of control EGFP.
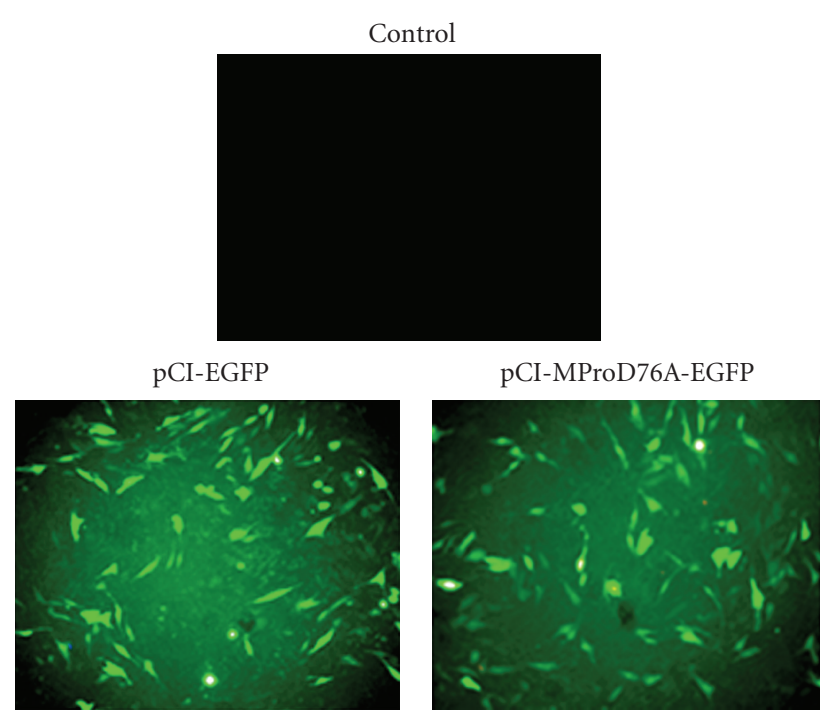

(a)

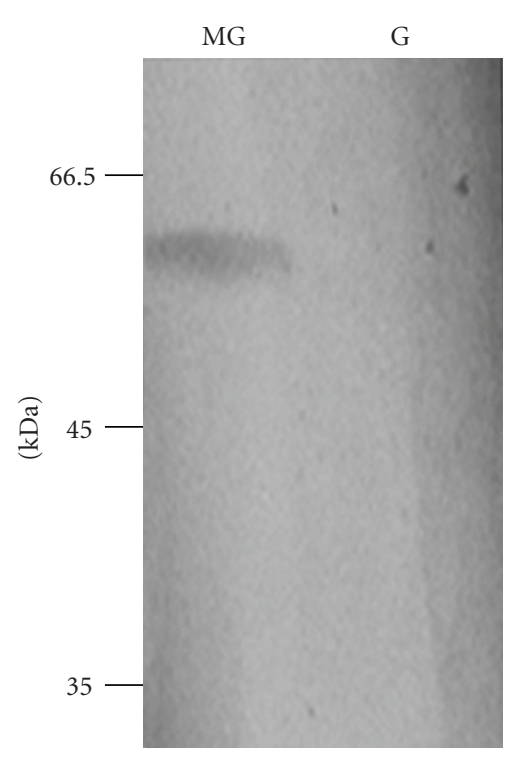

(b)

Figure 2: Expression of MProD76A-EGFP and EGFP in transiently transfected C2C12 cells. (a) Fluorescent microscopy of C2C12 cells transfected with pCI-MProD76A-EGFP or pCI-EGFP and untransfected control. (b) Western blot analysis of MProD76A-EGFP or EGFP secreted into the conditioned media. MG, pCI-MProD76A-EGFP-transfected cells; G, pCI-EGFP-transfected cells.

2.6. Histological and Morphometric Analysis. Cryosections $(8-10 \mu \mathrm{m})$ of gastrocnemius muscle were prepared from frozen muscles. Transverse sections of gastrocnemius were processed for hematoxylin and eosin (H\&E) staining. For morphometric analysis, images were acquired and the muscle fiber sizes were measured with Scion Image software.

2.7. Statistical Analysis. All statistical analyses were performed with SPSS statistical software. Values shown in the figures are the mean \pm SEM. All statistical tests were considered to be statistically significant at $P<.05$.

\section{Results}

3.1. pCI-MProD76A-EGFP and pCI-EGFP Vector Construction. To obtain a mutant myostatin propeptide that would have a more robust inhibiton effect on myostatin [19], we mutated the propeptide at the proteolytic cleavage site by replacing the aspartate at position 76 with an alanine residue (D76A). In addition, we fused EGFP to the C terminus of the mutant propeptide to monitor the expression and secretion of the transgene. This MProD76A-EGFP fusion construct was inserted into pCIneo vector under control of the cytomegalovirus (CMV) promoter to obtain the pCIMProD76A-EGFP vector (Figure 1). A vector pCI-EGFP was used as a control.

3.2. Expression of Mutant Propeptide in C2C12 Cells. In vitro expression of pCI-MProD76A-EGFP or pCI-EGFP was first examined in transiently transfected $\mathrm{C} 2 \mathrm{C} 12$ cells by EGFP fluorescence (Figure 2(a)). After transfection, C2C12 cells were placed into differentiation medium for $72 \mathrm{~h}$ to induce 

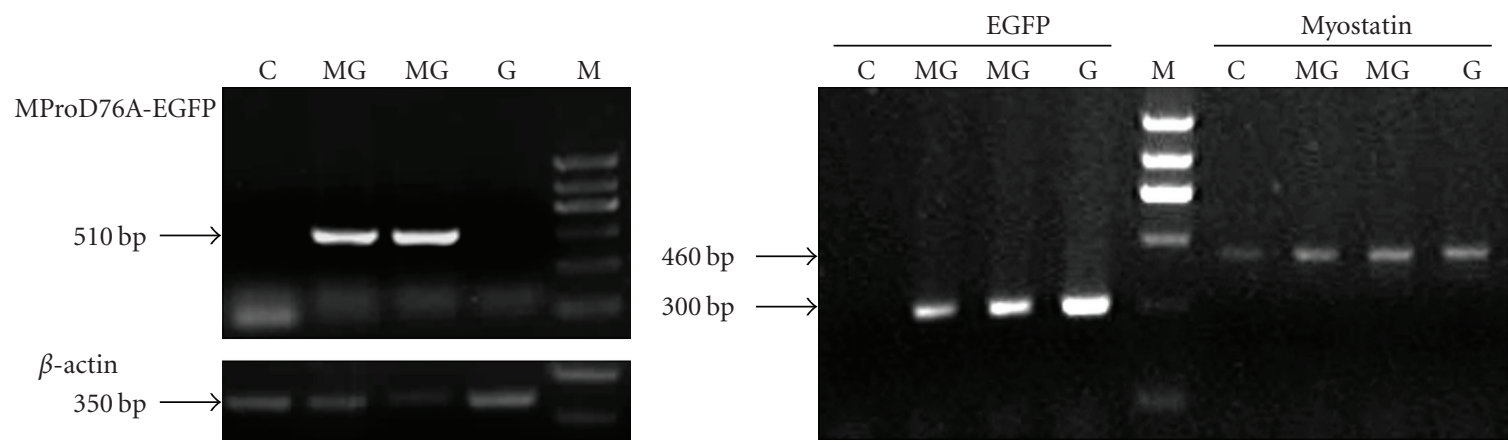

(a)

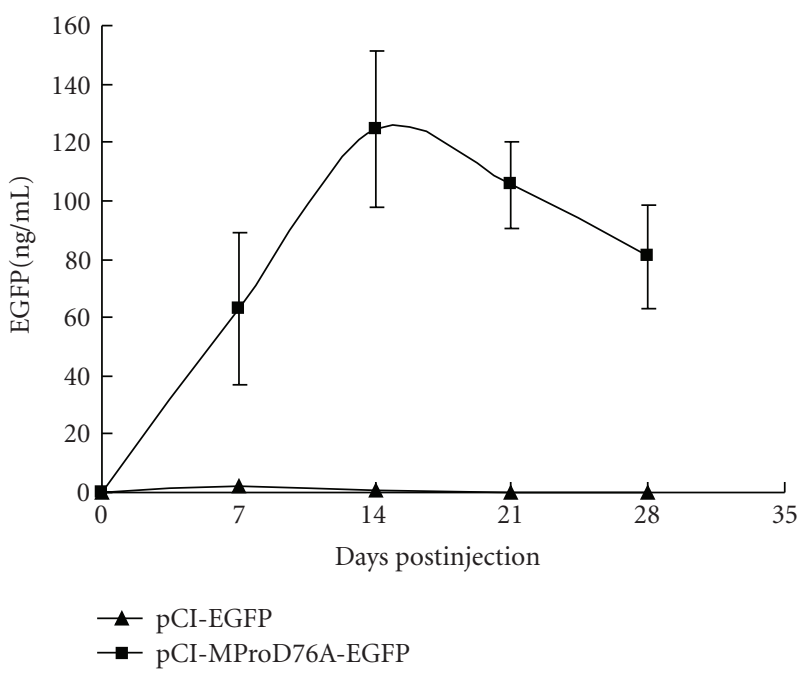

(b)

FIgURe 3: In vivo expression of pCI-MProD76A-EGFP in injected mice. (a) Expression of pCI-MProD76A-EGFP assayed by RT-PCR after intramuscular injection of pCI-MProD76A-EGFP into the quadriceps muscle of mice. Left panels: RT-PCR bands (510 bp) for MProD76AEGFP; RT-PCR bands (350 bp) for $\beta$-actin, which served as an internal control. Right panels: RT-PCR bands for EGFP (300 bp) and endogeous myostatin (460 bp). M, DNA markers; C, control muscle; MG, pCI-MProD76A-EGFP-injected muscle; G, pCI-EGFP-injected muscle. (b) The serum levels of the EGFP protein $(\mathrm{ng} / \mathrm{mL})$ in mice injected with pCI-MProD76A-EGFP or pCI-EGFP. The results are presented as means \pm SEM, $n=6$.

the cells to differentiate. The conditioned serum-free media and cells were harvested after an additional $48 \mathrm{~h}$ growth in serum-free medium. The mRNA expression of MProD76AEGFP or EGFP was confirmed by RT-PCR in C2C12 cells transfected with pCI-MProD76A-EGFP or pCI-EGFP (data not shown).

Secreted MProD76A-EGFP was detected by performing Western blot analysis of the collected conditioned media from pCI-MProD76A-EGFP or pCI-EGFP transfected $\mathrm{C} 2 \mathrm{C} 12$ cells (Figure 2(b)). MProD76A-EGFP was successfully detected in the conditioned media from pCIMProD76A-EGFP transfected C2C12 cells. EGFP cannot be secreted into media and therefore not detected in the conditioned media from pCI-EGFP transfected C2C12 cells. Thus, the skeletal muscle fibers transfected with pCIMProD76A-EGFP can express and secrete MProD76A-EGFP.

3.3. Effect of Mutant Propeptide on Normal Mice. To determine whether direct injection of plasmid DNA encoding mutant propeptide into muscle can enhance animal growth, four-week-old mice were anesthetized and injected with pCI-MProD76A-EGFP or pCI-EGFP into the regenerating quadriceps muscle. The in vivo expression of pCIMProD76A-EGFP and pCI-EGFP was confirmed by RT-PCR on injected muscle (Figure 3(a)). In addition, we quantified EGFP in blood from the injected animals (Figure 3(b)). A significant amount of MProD76A-EGFP was detected in blood samples from pCI-MProD76A-EGFP injected group, indicating that the mutant propeptide can be substantially expressed and secreted into the blood circulation from the site of muscle injection. However, EGFP is not a secreted protein and therefore was not detected in blood samples from pCI-EGFP injected group.

Mutant propeptide secreted into the blood circulation after intramuscular injection of pCI-MProD76A-EGFP significantly promoted growth throughout the whole body (Figure 4(a)), as shown by significant differences in body weight at 30 days $(29.83 \pm 0.60 \mathrm{~g}$ versus $26.95 \pm 0.35 \mathrm{~g}$, 


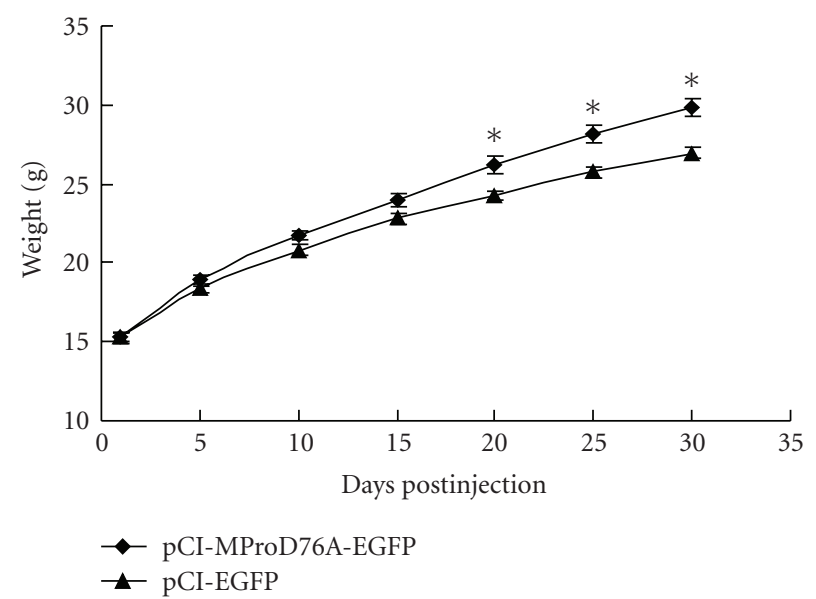

(a)

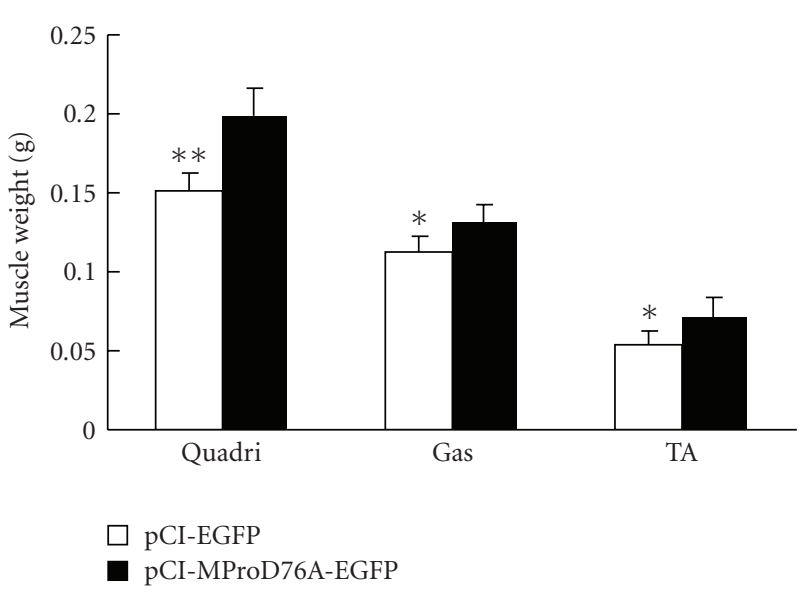

(b)

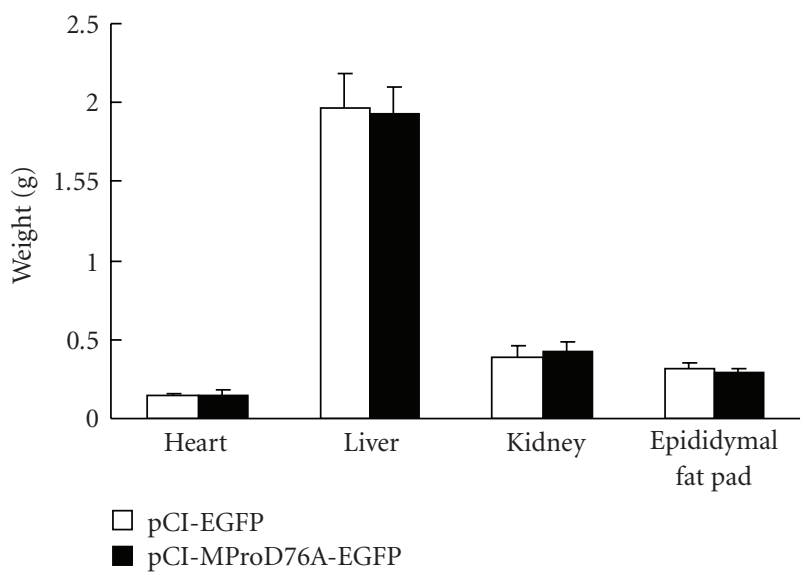

(c)

FIGURE 4: Effects of myostatin propeptide delivery on growth performance of mice. $(n=6)$. Four-week-old mice were injected with pCIMProD76A-EGFP or pCI-EGFP into the regenerating quadriceps muscle. (a) Mice injected with pCI-MProD76A-EGFP showed a significant increase in body mass compared with pCI-EGFP control mice $\left({ }^{*} P<.05\right)$. (b) The mass of individual hindlimb muscles is increased in mice injected with pCI-MProD76A-EGFP $\left({ }^{* *} P<.01\right.$; $\left.{ }^{*} P<.05\right)$. (c) Comparison of the heart, the liver, the kidney, and the epididymal fat pad weights, no significant difference was found between the two groups.

$P<.05)$ after injection of pCI-MProD76A-EGFP or pCIEGFP. We also dissected and weighed the epididymal fat pad, the heart, the kidney, the liver, and the individual muscles including the quadriceps (Quadri), gastrocnemius (Gas), tibialis anterior (TA) at the end point of the experiment. All the examined skeletal muscles (TA, Gas, Quadri, and Diaph) showed a significant increase in muscle mass for pCI-MProD76A-EGFP injected group (Figure 4(b)). The quadriceps muscle, which showed the greatest increase among the muscles examined, displayed a $31.58 \%$ increase in muscle mass over the control group $(P<.01)$. However, the weights of the epididymal fat pad, the heart, the kidney, and the liver did not show significant difference between the two groups (Figure $4(\mathrm{c})$ ).

To determine whether the increase in muscle mass resulted from hypertrophy or hyperplasia, we performed histological and morphometric analysis of gastrocnemius muscle. H\&E staining showed larger myofiber sizes in the pCI-MProD76A-EGFP injected mice (Figure 5(a)). Digital morphometric analysis of 300 myofibers from each of three mice injected with pCI-MProD76A-EGFP revealed a significant increase in myofiber diameter compared with control mice (Figure 5(b)). The diameter of $77 \%$ pCIMProD76A-EGFP treated myofibers was measured at 30$60 \mu \mathrm{m}$, whereas that of $86 \%$ control myofibers was measured at $15-45 \mu \mathrm{m}$. There was a shift in muscle size toward bigger myofiber diameter range. But no significant difference was found in the total muscle myofiber numbers per transverse sections between the two groups (data not shown). These results suggested that increased muscle mass was due to hypertrophy rather than hyperplasia.

\section{Disscussion}

The study on myostatin function has raised the possibility that myostatin inhibition may be useful for increasing muscle mass for agricultural applications. In this regard, inhibition of endogenous myostatin has been shown to have beneficial 
pCI-EGFP
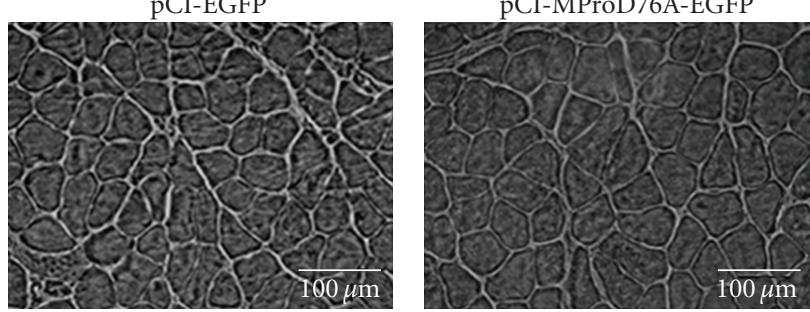

(a)

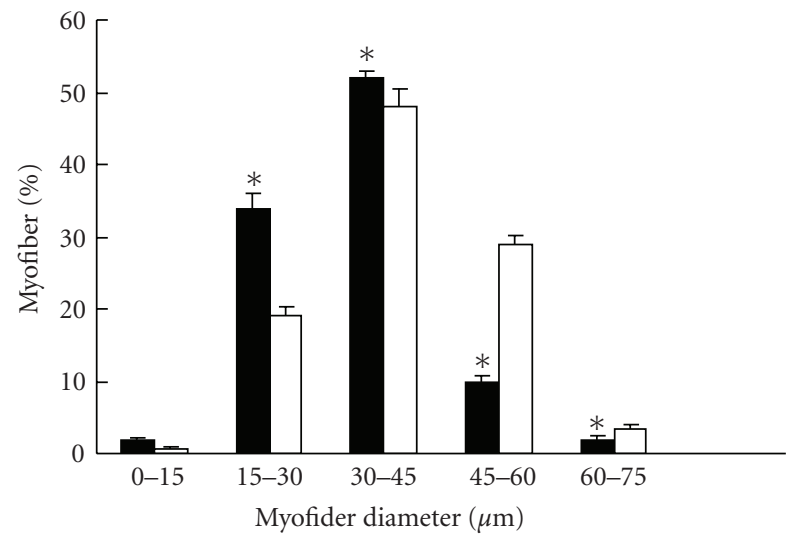

pCI-EGFP

pCI-MProD76A-EGFP

(b)

FIGURE 5: Morphometric analysis of gastrocnemius muscle. (a) $\mathrm{H} \& \mathrm{E}$ staining displays myofiber hypertrophy in muscles injected with pCI-MProD76A-EGFP compared with pCI-EGFP control, Scale bars: $100 \mu \mathrm{m}$. (b) Distribution of muscle fiber sizes in mice injected with either pCI-MProD76A-EGFP or pCI-EGFP. The diameter of $77 \%$ pCI-MProD76A-EGFP treated myofibers was measured at $30-60 \mu \mathrm{m}$, whereas that of $86 \%$ control myofibers was measured at $15-45 \mu \mathrm{m}\left({ }^{*} P<.05, n=3\right)$.

effects on the animal growth performance, such as by intraperitoneal injections of recombinant myostatin binding proteins into mice $[4,24]$, or various fish $[25]$ and producing transgenic animals expressing higher levels of myostatin inhibitors [9-12]. However, limitations of using recombinant myostatin binding proteins are the high cost of these proteins and the requirement for frequent administration (1-2 times per week) of intraperitoneal injections. The transgenic animal-based strategy is involved in the genetic alteration of genome and is not practical for livestock breeding and production. Using plasmid-mediated delivery of myostatin inhibitor, it might be possible to inhibit animal endogenous myostatin in a much simpler and less expensive manner than other strategies.

We tested the inhibition of myostatin by plasmidmediated expression of a mutant propeptide in normal mice. A significant increase in skeletal muscle mass was obtained after myostatin inhibition. The increased muscle mass was found in the injected hindlimb muscles and remote muscles to the injection site. Thus, the mutant propeptide produced in injected muscle was secreted into the blood circulation, enhancing muscle growth throughout the whole body. However, when inhibition of myostatin by mutant propeptide is long-term occurrence in the whole body, there are still some concern that immune response and adverse effects on gonadal function and meat quality may be caused by the mutant propeptide. Additional experiments would be needed to explore these before the widespread use of such strategy.

We further revealed that the increase in muscle mass with MProD76A plasmid injection was the result of muscle fiber hypertrophy and not hyperplasia. This finding differs from observations of myostatin gene knockout mice and $\mathrm{Mstn}^{\mathrm{D} 76 \mathrm{~A} / \mathrm{D} 76 \mathrm{~A}}$ mice, which had both hypertrophy and hyperplasia $[2,26]$. Conversely, neutralizing antibodies to myostatin causes significant increases in muscle mass when administered to adult mice, and this increase in muscle growth appears to result from muscle fiber hypertrophy [13]. Thus, the development of hyperplasia versus hypertrophy is likely to be dependent on the time during embryonic development or postnatal life at which myostatin activity is lost [1]. It is noteworthy that systemic myostatin inhibition did not cause cardiac muscle hypertrophy despite skeletal muscle hypertrophy, suggesting that cardiac muscle hypertrophy may not be a concern for application of plasmid-mediated delivery of myostatin propeptide. In addition, we did not observe a significant difference in epididymal fat pad mass after myostatin inhibition. This result differs from the results reported in the previous papers where fat accumulation is decreased in the absence of myostatin [27, 28]. This discrepancy could be due to differences in loss of myostatin expression during development and adulthood [13].

In this study, EGFP was fused with MProD76A to monitor the expression and secretion of transgene. But an immune response may be elicited in the injected animal, which potentially lowers the expression of transgene. It is likely that further optimization of our vector system will be necessary for practical application. Wolfman have reported that the propeptide fused with IgG Fc domain may prolong its half-life of circulation in vivo [19]. Amino acids mutation of the propeptide that are known to be sites for different peptidases could also prolong its half-life of circulation in vivo. In addition, synthetic muscle-specific promoter and gene delivery technique like electroporation have been shown to effectively increase the utility for delivery of therapeutic proteins in pig $[29,30]$ and $\operatorname{dog}[31]$. These methods would improve gene expression and transfection efficiency of plasmid DNA encoding MProD76A when applied in farm animal.

In summary, our studies have demonstrated the effectiveness of myostatin inhibition by plasmid-mediated delivery of the mutant myostatin propeptide in mice. Moreover, the low cost, the stability, the possibility of mass production of plasmid DNA as well as the ease of storage and operation, allow the development of this strategy not only in livestock production but also as a potential treatment for human muscle wasting diseases, including sarcopenia and muscular dystrophies. However, owing to the larger animal size and longer growth periods, this strategy still needs further investigation in large animal models. 


\section{Acknowledgments}

The authors thank Yan Ren and Guoqing Du for their assistance. This work was supported by a grant from the Bingtuan Doctor Foundation Program (008JC06, Xinjiang, China).

\section{References}

[1] S.-J. Lee, "Regulation of muscle mass by myostatin," Annual Review of Cell and Developmental Biology, vol. 20, pp. 61-86, 2004.

[2] A. C. McPherron, A. M. Lawler, and S.-J. Lee, "Regulation of skeletal muscle mass in mice by a new TGF- $\beta$ superfamily member," Nature, vol. 387, no. 6628, pp. 83-90, 1997.

[3] L. Grobet, L. J. R. Martin, D. Poncelet, et al., "A deletion in the bovine myostatin gene causes the double-muscled phenotype in cattle," Nature Genetics, vol. 17, no. 1, pp. 71-74, 1997.

[4] R. Kambadur, M. Sharma, T. P. L. Smith, and J. J. Bass, "Mutations in myostatin (GDF8) in double-muscled Belgian Blue and Piedmontese cattle," Genome Research, vol. 7, no. 9, pp. 910-916, 1997.

[5] A. C. McPherron and S.-J. Lee, "Double muscling in cattle due to mutations in the myostatin gene," Proceedings of the National Academy of Sciences of the United States of America, vol. 94, no. 23, pp. 12457-12461, 1997.

[6] A. Clop, F. Marcq, H. Takeda, et al., "A mutation creating a potential illegitimate microRNA target site in the myostatin gene affects muscularity in sheep," Nature Genetics, vol. 38, no. 7, pp. 813-818, 2006.

[7] D. S. Mosher, P. Quignon, C. D. Bustamante, et al., "A mutation in the myostatin gene increases muscle mass and enhances racing performance in heterozygote dogs," PLoS Genetics, vol. 3, no. 5, pp. 779-786, 2007.

[8] M. Schuelke, K. R. Wagner, L. E. Stolz, et al., "Myostatin mutation associated with gross muscle hypertrophy in a child," The New England Journal of Medicine, vol. 350, no. 26, pp. 2682-2688, 2004.

[9] X. Zhu, M. Hadhazy, M. Wehling, J. G. Tidball, and E. M. McNally, "Dominant negative myostatin produces hypertrophy without hyperplasia in muscle," FEBS Letters, vol. 474, no. 1, pp. 71-75, 2000.

[10] S.-J. Lee and A. C. McPherron, "Regulation of myostatin activity and muscle growth," Proceedings of the National Academy of Sciences of the United States of America, vol. 98, no. 16, pp. 9306-9311, 2001.

[11] C. Xu, G. Wu, Y. Zohar, and S.-J. Du, "Analysis of myostatin gene structure, expression and function in zebrafish," Journal of Experimental Biology, vol. 206, no. 22, pp. 4067-4079, 2003.

[12] J. Acosta, Y. Carpio, I. Borroto, O. González, and M. P. Estrada, "Myostatin gene silenced by RNAi show a zebrafish giant phenotype," Journal of Biotechnology, vol. 119, no. 4, pp. 324$331,2005$.

[13] L.-A. Whittemore, K. Song, X. Li, et al., "Inhibition of myostatin in adult mice increases skeletal muscle mass and strength," Biochemical and Biophysical Research Communications, vol. 300, no. 4, pp. 965-971, 2003.

[14] R. Draghia-Akli, M. L. Fiorotto, L. A. Hill, P. Brandon Malone, D. R. Deaver, and R. J. Schwartz, "Myogenic expression of an injectable protease-resistant growth hormonereleasing hormone augments long-term growth in pigs," Nature Biotechnology, vol. 17, no. 12, pp. 1179-1183, 1999.
[15] J. A. Wolff, R. W. Malone, P. Williams, et al., "Direct gene transfer into mouse muscle in vivo," Science, vol. 247 , no. 4949 , pp. 1465-1468, 1990.

[16] J. A. Wolff, J. J. Ludtke, G. Acsadi, P. Williams, and A. Jani, "Long-term persistence of plasmid DNA and foreign gene expression in mouse muscle," Human Molecular Genetics, vol. 1, no. 6, pp. 363-369, 1992.

[17] H. L. Davis, R. G. Whalen, and B. A. Demeneix, "Direct gene transfer into skeletal muscle in vivo: factors affecting efficiency of transfer and stability of expression," Human Gene Therapy, vol. 4, no. 2, pp. 151-159, 1993.

[18] S. K. Tripathy, E. C. Svensson, H. B. Black, et al., "Long-term expression of erythropoietin in the systemic circulation of mice after intramuscular injection of a plasmid DNA vector," Proceedings of the National Academy of Sciences of the United States of America, vol. 93, no. 20, pp. 10876-10880, 1996.

[19] N. M. Wolfman, A. C. McPherron, W. N. Pappano, et al., "Activation of latent myostatin by the BMP-1/tolloid family of metalloproteinases," Proceedings of the National Academy of Sciences of the United States of America, vol. 100, no. 26, pp. 15842-15846, 2003.

[20] S. Bogdanovich, K. J. Perkins, T. O. B. Krag, L.-A. Whittemore, and T. S. Khurana, "Myostatin propeptide-mediated amelioration of dystrophic pathophysiology," FASEB Journal, vol. 19, no. 6, pp. 543-549, 2005.

[21] I. Danko, J. D. Fritz, S. Jiao, K. Hogan, J. S. Latendresse, and J. A. Wolff, "Pharmacological enhancement of in vivo foreign gene expression in muscle," Gene Therapy, vol. 1, no. 2, pp. 114-121, 1994.

[22] G. S. MacColl, F. J. Novo, N. J. Marshall, M. Waters, G. Goldspink, and P. M. G. Bouloux, "Optimisation of growth hormone production by muscle cells using plasmid DNA," Journal of Endocrinology, vol. 165, no. 2, pp. 329-336, 2000.

[23] S.-H. Seok, M.-W. Baek, H.-Y. Lee, et al., "Quantitative GFP fluorescence as an indicator of arsenite developmental toxicity in mosaic heat shock protein 70 transgenic zebrafish," Toxicology and Applied Pharmacology, vol. 225, no. 2, pp. 154 161, 2007.

[24] S.-J. Lee, L. A. Reed, M. V. Davies, et al., "Regulation of muscle growth by multiple ligands signaling through activin type II receptors," Proceedings of the National Academy of Sciences of the United States of America, vol. 102, no. 50, pp. 18117-18122, 2005.

[25] Y. Carpio, J. Acosta, R. Morales, Y. Santisteban, A. Sanchez, and M. P. Estrada, "Regulation of body mass growth through activin type IIB receptor in teleost fish," General and Comparative Endocrinology, vol. 160, no. 2, pp. 158-167, 2009.

[26] S.-J. Lee, "Genetic analysis of the role of proteolysis in the activation of latent myostatin," PLOS ONE, vol. 3, no. 2, article e1628, 2008.

[27] A. C. McPherron and S.-J. Lee, "Suppression of body fat accumulation in myostatin-deficient mice," Journal of Clinical Investigation, vol. 109, no. 5, pp. 595-601, 2002.

[28] J. Lin, H. B. Arnold, M. A. Della-Fera, M. J. Azain, D. L. Hartzell, and C. A. Baile, "Myostatin knockout in mice increases myogenesis and decreases adipogenesis," Biochemical and Biophysical Research Communications, vol. 291, no. 3, pp. 701-706, 2002.

[29] R. Draghia-Akli, K. M. Ellis, L. A. Hill, P. B. Malone, and M. L. Fiorotto, "High-efficiency growth hormone-releasing hormone plasmid vector administration into skeletal muscle mediated by electroporation in pigs," The FASEB Journal, vol. 17, no. 3, pp. 526-528, 2003. 
[30] R. Draghia-Akli and M. L. Fiorotto, "A new plasmid-mediated approach to supplement somatotropin production in pigs," Journal of Animal Science, vol. 82, pp. E264-E269, 2004.

[31] R. Draghia-Akli, K. K. Cummings, A. S. Khan, P. A. Brown, and R. H. Carpenter, "Effects of plasmid-mediated growth hormone releasing hormone supplementation in young, healthy Beagle dogs," Journal of Animal Science, vol. 81, no. 9, pp. 2301-2310, 2003. 

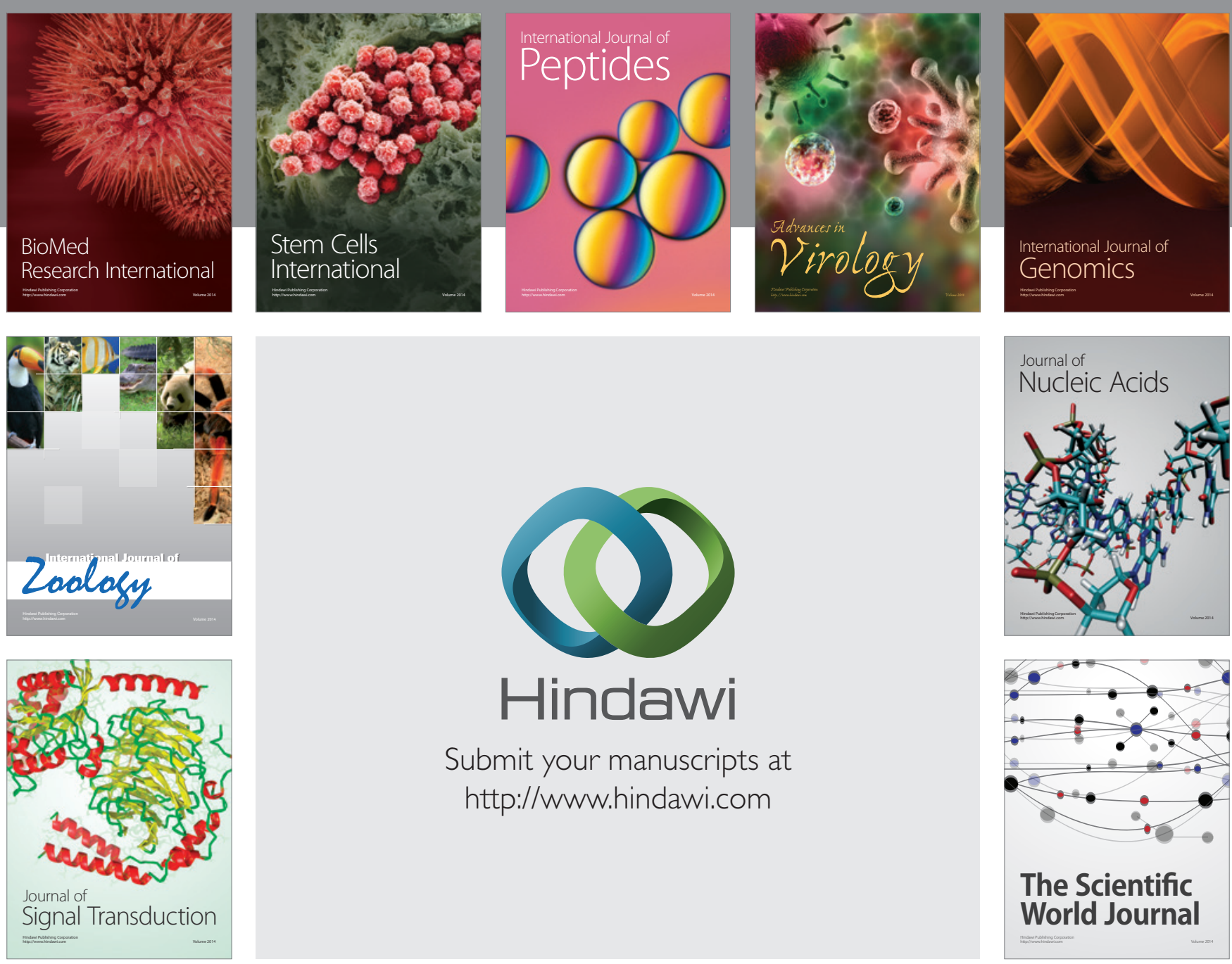

Submit your manuscripts at

http://www.hindawi.com
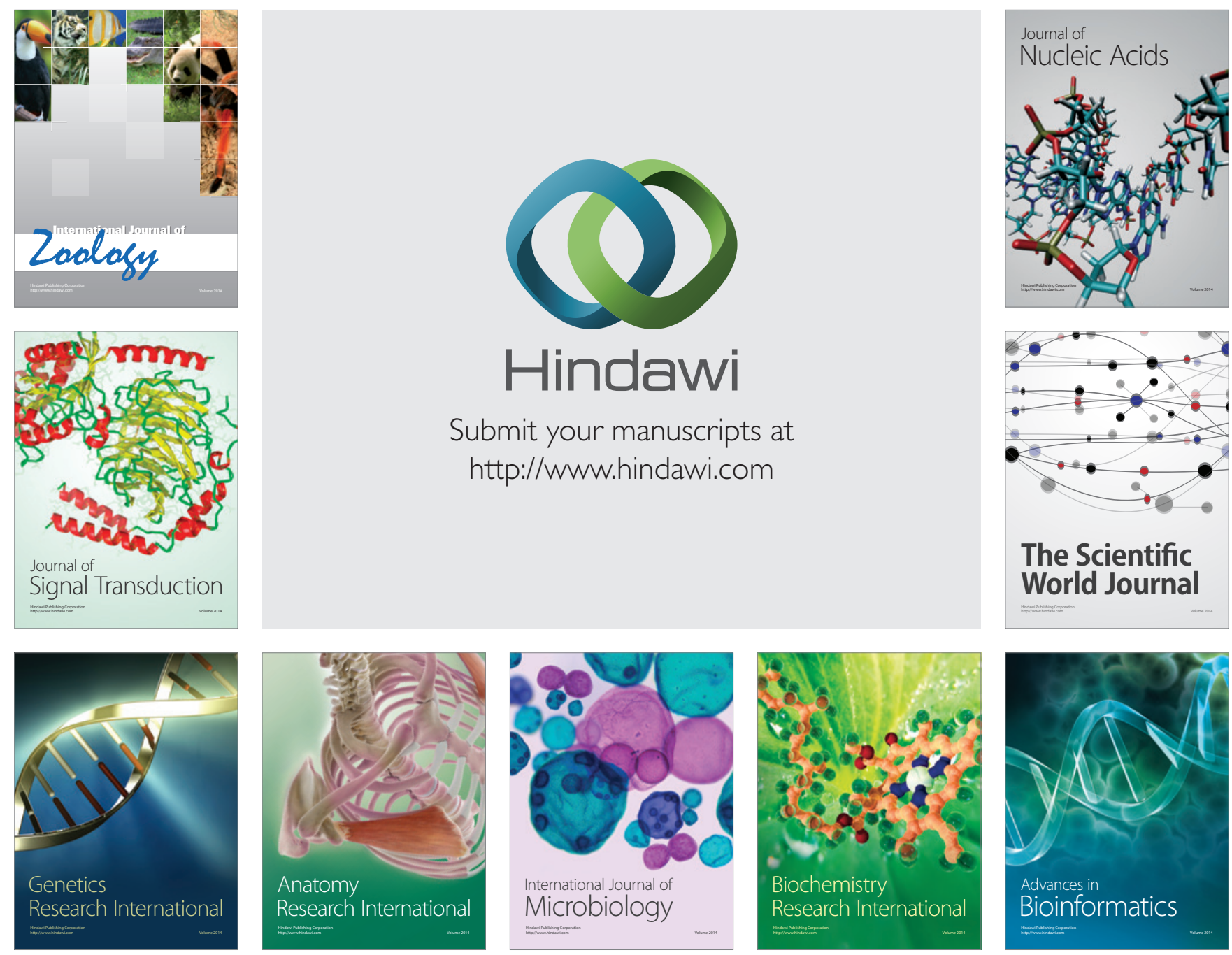

The Scientific World Journal
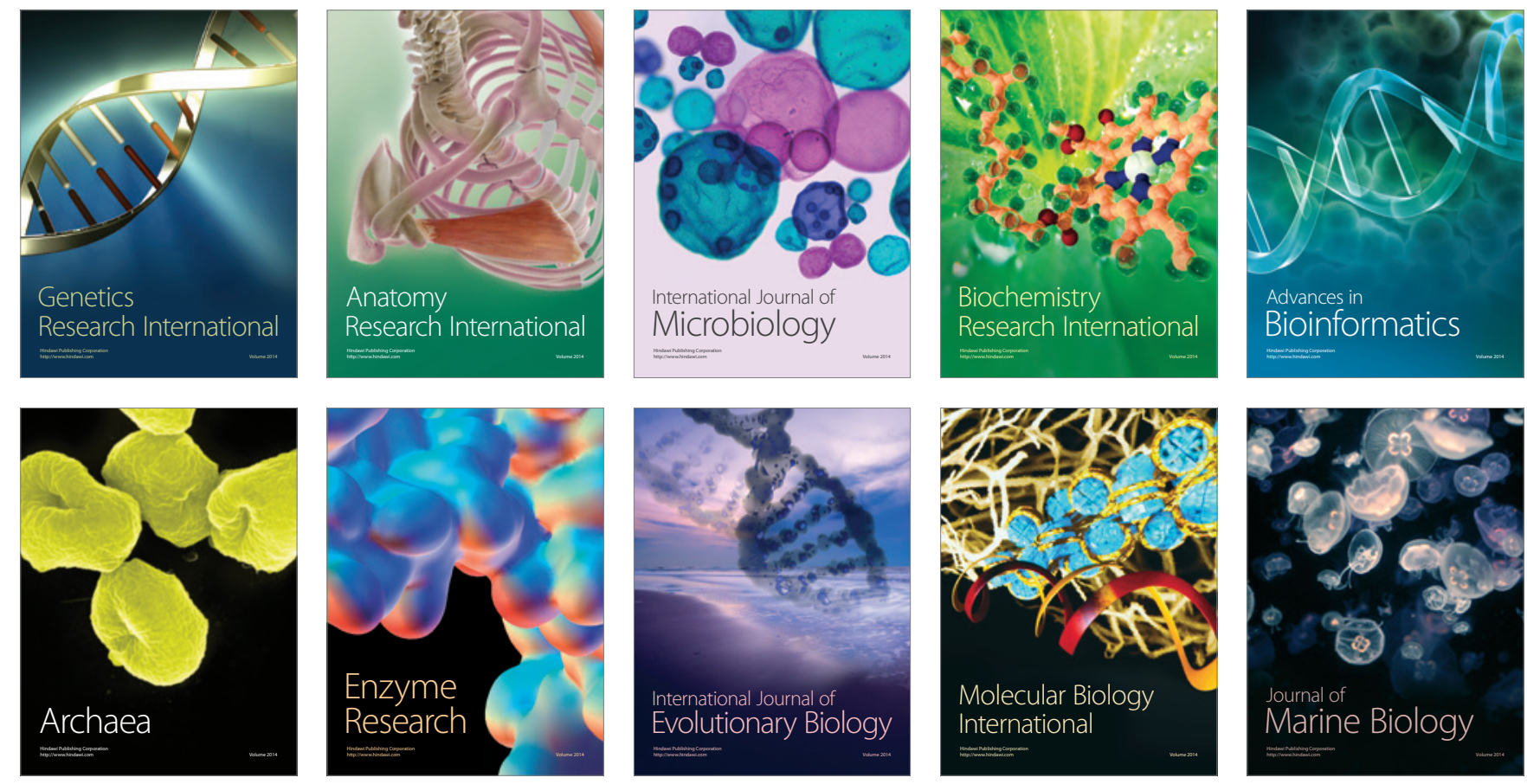